\title{
Clinical features and neuroimaging (CT and MRI) findings in presumed Zika virus related congenital infection and microcephaly: retrospective case series study
}

This Research paper (BMJ 2016;353:i1901, doi:10.1136/bmj. i1901) contains an error in table 5 . The number (\%) of children with calcifications in the cerebellum should read " 2 (9.1)," instead of "11 (50)" as was published. 